\title{
Variations
}

Variations

Revue internationale de théorie critique

$17 \mid 2012$

Critique du travail

\section{Des pinoches dans la coque}

\section{Claire Flécher}

\section{(2) OpenEdition \\ Journals}

Édition électronique

URL : http://journals.openedition.org/variations/375

DOI : 10.4000/variations.375

ISSN : 1968-3960

\section{Éditeur}

Les amis de Variations

\section{Référence électronique}

Claire Flécher, «Des pinoches dans la coque », Variations [En ligne], 17 | 2012, mis en ligne le 15

octobre 2012, consulté le 19 avril 2019. URL : http://journals.openedition.org/variations/375 ; DOI :

$10.4000 /$ variations.375

Ce document a été généré automatiquement le 19 avril 2019

Les ami•e•s de Variations 


\title{
Des pinoches dans la coque
}

\author{
Claire Flécher
}

1 Voilà déjà dix jours que j'attendais que le téléphone sonne afin de connaître la date et le lieu de l'embarquement. Dix jours d'attente, le sac prêt et le corps sur le qui-vive, où il est bien difficile de planifier quoi que ce soit, tant il faut se tenir prêt à partir dans l'heure qui suit. La seule certitude, est que le bateau est un pétrolier fonctionnant au tramping.

2 Le tramping. Cet élément est important : il est, en grande partie, la source de toute cette incertitude et, comme je le verrai une fois à bord, une donnée structurante de l'organisation et des outils de travail à bord. En effet, à la différence des liners qui effectuent toujours les mêmes trajets, les navires exploités au tramping ne savent jamais qu'au dernier moment la prochaine destination car ils ne sont jamais affectés au même affréteur ${ }^{1}$, lequel change au hasard des appels d'offre.

En cinq minutes on peut faire demi- tour. Hop un coup de frein à main et on part charger en Libye ! Y'a jamais rien de fixe. Quand tu embarques tu sais jamais quand tu débarques, tu sais jamais où tu vas, quand tu vas dormir, quand tu vas te lever, quand tu vas manger... (Chef mécanicien) ${ }^{2}$.

3 Dans ce type d'exploitation, il est en effet possible de charger un produit acheté par un client, qui sera revendu au cours du voyage. Nous sommes ici dans du juste à temps en lien direct avec les évolutions du cours boursier de la matière concernée.

Et un jour le téléphone sonne. C'est le service de l'armement. Ils m'ont réservé un avion dans deux jours, direction l'Italie, où un taxi m'attendra pour me conduire jusqu'au navire. Celui-ci vient de se mettre à quai pour l'opération commerciale. Pour rejoindre le navire, il faut environ une heure de route nationale. Néanmoins, rien à voir avec le port de Rotterdam, où il nous avait fallu plus d'une heure pour ne traverser que la zone portuaire, imbroglio sans fin d'autoroutes, de ponts, et de tunnels. Une ville dans la ville. Ici, le port est tout petit, les services de contrôle très restreints. À Rotterdam, en conformité avec le code ISPS ${ }^{3}$, il m'avait fallu signer plusieurs papiers, montrer patte blanche et passeport biométrique. Ici, rien de tout cela, je passe à ma grande surprise le portail sans me faire arrêter, et monte de mon propre chef à bord.

Le navire transporte du diesel et de l'essence. Le pont est ainsi un champ de tuyaux et de vannes de différentes tailles, ce qui est plutôt pas mal pour la santé des navigants car ces 
marchandises sont très volatiles. Alors qu'avant, il fallait ouvrir les cuves pour vérifier le niveau, tout est à présent contrôlé automatiquement et à distance. C'est donc à $100 \%$ derrière les écrans que se surveille le chargement. Pas la moindre trace de pétrole à l'horizon lorsque l'on vient sur le pont. C'est tout propre, tout net, on est bien loin de la poussière du minerais de fer à bord du vraquier qui pénètre tous les pores de la peau. Le produit est là mais on ne le voit, ne le touche, et à présent, ne le respire, jamais.

6 Je monte donc à la coupée, où je me fais réceptionner par un matelot chargé de vérifier les allées et venues à bord qu'il consigne dans un cahier, le visitor log book. Celui-ci me conduit directement au château où je trouve l'officier de quart au poste de contrôle (PC) cargo devant des ordinateurs qui indiquent la cadence du chargement en cours. Je suis prise en charge par le capitaine qui m'amène à ce qui sera ma cabine pour les prochaines semaines. C'est l'heure du déjeuner, nous redescendons donc directement au carré où sont servis les repas.

7 Le temps de l'opération commerciale (OC) est un temps de travail très intensif, où chaque marin est assigné à une mission dont il faut s'acquitter au plus vite afin de ne pas retarder le moment du départ. Un navire à quai coûte cher, et nécessite toute une logistique mise en œuvre par le port afin de produire les divers services dont le navire a besoin lors de l'escale (approvisionnement, soutage, évacuation des déchets, pilotage).

8 Lors de l'OC, il faut veiller en continu à ce que le chargement se déroule comme prévu par le second capitaine, lequel est responsable de toute l'opération : en amont avec le calcul des cadences; pendant le chargement/déchargement des produits (opération dans laquelle il est secondé par les lieutenants pont grâce au système de quart, ainsi que par le pompiste, ouvrier spécialisé chargé de vérifier les branchements des tuyaux); et enfin pour tout le côté administratif des papiers à signer avec les multiples acteurs et commanditaires concernés par la marchandise. Le service machine, responsable quant à lui de l'autonomie énergétique du navire, doit prévoir et anticiper - en tenant compte des contraintes de cette navigation au tramping - les commandes de carburants (le soutage), de nourriture, de pièces de rechange pour la maintenance, mais aussi l'évacuation des boues ou des déchets non éliminables à bord. Enfin, le capitaine doit recevoir les visiteurs et contrôleurs de toute espèce, signer les papiers du port, du client, de l'armateur, tout en supervisant ce qui se passe sur le pont. Le temps de l'opération commerciale constitue donc un pic d'intensivité dans l'activité. Lorsque le navire fait escale tous les deux jours, les rythmes de travail sont particulièrement éprouvants.

9 À bord, vingt et une personnes se répartissent entre les deux services : le pont et la machine. Au pont, trois lieutenants navigation font le quart à tour de rôle, et le second capitaine est chargé des $O C$ et de l'entretien du navire. Sous ses ordres, le bosco et trois matelots. Parce que nous sommes ici sur un pétrolier, il existe également un pompiste qui, hors OC, seconde le bosco et le second capitaine dans leur travail. Il est chargé de vérifier vannes et tuyaux par où passe le produit. À la machine, il existe deux monteurs ( fitter), un nettoyeur, un électricien, et enfin trois officiers (le troisième mécanicien, le second mécanicien, le chef mécanicien). Une élève officier, une Zef, est également à bord, conformément à la formation délivrée dans les écoles maritimes françaises qui oblige un temps de navigation minimum afin de valider le diplôme obtenu à l'école. Last but not least , la gargouille, service très important dirigé par le cuisinier et l'aide cuisinier, lequel est également chargé du service et du ménage. Le commandant chapeaute les deux services : s'il est davantage présent à la passerelle, il est responsable en dernière instance de toute l'expédition maritime. 


\section{Dis-moi d'où tu viens, je te dirais combien je te paye}

10

à cause des contrats d'affrètement que le navire est supposé remplir pour le mois qui arrive. Avant eux, tous les matelots étaient philippins, mais comme le navire semble être destiné à rester en Europe pour faire du cabotage pour un moment, la compagnie doit se plier aux normes d'emploi européennes qui stipulent qu'un navire faisant du cabotage le long des côtes européennes, doit employer une main-d'œuvre européenne. Donc du jour au lendemain, l'équipage a été entièrement renouvelé : les philippins renvoyés chez eux, et les lettons embarqués à bord.

11 Ces derniers sont embauchés au contrat pour une durée de quatre mois, alors que les matelots philippins étaient sous un régime de contrats de six mois. Dans d'autres compagnies, cela peut aller jusqu'à un an renouvelable. Embauchés en free-lance, les matelots reçoivent des salaires élevés comparés au salaire moyen de leur pays d'origine. Néanmoins, ces derniers n'ont pas accès aux institutions salariales d'une part, et d'autre part, ne sont pas dans une relation d'emploi stable. Les officiers français, eux, sont salariés en CDI ou CDD, avec un régime de deux ou trois mois embarqués, suivi de deux ou trois mois de congés. Pour eux, un jour travaillé équivaut alors à un jour de congé. Sur ce même lieu de travail se côtoient donc différents statuts, et cette gestion différenciée est directement liée à la nationalité du marin. La logique présidant à ce mode de gestion est identique à celle poussant les entreprises à délocaliser leurs services dans des pays où le coût du travail est plus faible. E. Terray parle de «délocalisation sur place $»^{4}$ pour caractériser des secteurs tels que la confection, le bâtiment ou la restauration dans lesquels il n'est pas possible de déplacer le lieu de production. Or, si sur un chantier, il est possible de distinguer trois grands types de régimes entre les ouvriers sans-papiers, les chefs de chantiers, souvent portugais, et les cadres français, un navire aura autant de statuts différents que de nationalités présentes ${ }^{5}$. Aussi, à niveau hiérarchique égal, deux marins peuvent être payés différemment, car leurs salaires respectifs sont calculés en fonction du niveau de vie de leur pays d'origine.

Alors que les marins salariés de la compagnie ont un lien direct avec l'armement, les matelots en free-lance sont gérés par ceux qui sont communément appelés des «marchands d'hommes» ou "manning agencies». Ces agences de gestion de main d'œuvre, situées dans des bassins d'emploi, sont chargées de vérifier les brevets des matelots et d'organiser les relèves. Ils sont des acteurs centraux de la mondialisation du marché du travail maritime. Si une compagnie se retrouve en pénurie d'officiers salariés, celle-ci fait appel à son agence qui lui trouvera rapidement un marin. Ce système participe par ailleurs à un chantage à l'emploi.

On a eu un Zef ici, il a quand même étalé. Il a fait trois mois ici, donc il était avec moi le dernier coup. Comme élève. Il a débarqué en Egypte. Il a eu douze jours de congés et ils l'ont rappelé et menacé que c'était ça ou rien. Donc il est reparti pour trois mois. En six mois, il aura eu dix jours de congés.

$\mathrm{Du}$ coup, s'ils ne trouvent aucun français qui accepte de rembarquer, ils font appel aux boites de manning en Roumanie ou autre?

Oui comme là à l'heure actuelle sur le 'Tonnerre'. Ils avaient pas de chef, y'en a pas mal qui ont arrêté qui sont partis. Qui ont démissionné même. Y’en a pas mal qui sont... qui ont un peu craqué et qui sont en arrêt. Donc ils ont pris un chef croate et trois semaines après on savait que le bateau allait être déflaggé.

Variations, 17| 2012 

pour l'inscrire dans des pays proposant une fiscalité intéressante. Souvent, les armateurs choisissent des pays tels que le Panama ou Chypre qui ont des capacités de contrôle de leur navire très réduites ${ }^{6}$. Côté droit du travail, le référentiel de l'armateur devient les conventions internationales adoptées par l'OMI, agence spécialisée de l'ONU, qui sont des mesures prises a minima afin de contenter un maximum d'acteurs. Cette opération est intéressante à plus d'un titre : outre les avantages fiscaux, elle permet de passer à travers un certain nombre de réglementations nationales, souvent plus contraignantes que celles internationales, tout en évitant les mailles du filet des contrôles sécurité très aléatoires. Elle permet en outre de travailler avec des agences de manning.

L'usage des marchands d'hommes s'inscrit dans une politique de réduction des coûts. Il permet à l'armateur d'externaliser sa gestion du personnel, et du même coup, de se dégager de toute responsabilité en cas, par exemple, de détention de faux brevets des matelots. Mais surtout, ce système instaure une relation d'emploi où le marin, afin de rendre régulier ses temps d'embarquements (et, partant, de congés), doit se montrer tout à fait disponible.

15 Gérés par ces intermédiaires, les matelots n'ont souvent pas d'autre choix que d'attendre que ces derniers les contactent. Un matelot letton me raconte ainsi que lors de ses " congés ", il lui est très difficile de se projeter car le téléphone est toujours branché : il faut être prêt à repartir du jour au lendemain si l'agence le contacte. Un refus de sa part se traduirait par un comportement sanction de la part du marchand d'homme qui ne le rappellera plus pendant quelques mois : "C'est que, quand tu dis non deux fois, ils te disent non aussi deux fois. » C'est pourquoi, pour certains, mieux vaut des contrats longs d'un an plutôt que de six mois : tant que l'on est à bord, le salaire est assuré7. Et comme on ne sait jamais quand et combien de temps il sera possible de réembarquer par la suite, c'est toujours ça de pris. Dans ce cadre, les matelots n'ont souvent aucun moyen de contacter les compagnies de leur propre initiative. Les agences font figure d'intermédiaire autant que de filtre entre les matelots et les compagnies. Ainsi, lors de l'embarquement, une demande collective de la part des Lettons a été faite auprès du commandant pour savoir s'il était possible de prendre contact directement avec celle du navire. Acceptant de transmettre cette revendication, la réponse obtenue par la compagnie fut on ne peut plus claire. Le non catégorique témoigne de la volonté ferme de conserver cet échelon intermédiaire entre elle et ces employés en free-lance.

Cette situation pose les marins en position de sujets déférents face à une terre qui possède le dernier mot et entretient par ce biais une relation de dépendance avec les employés. Dépossédés de toute information et de tout moyen de faire appel, ils n'ont alors d'autre choix que d'attendre, et de se plier aux propositions que l'agence leur fait. Il s'agit alors de prouver que l'on est disponible, en acceptant les offres d'embarquement alors même que l'on vient juste de rentrer de mer, dans l'espoir d'une éventuelle stabilisation de la relation d'emploi ou, au mieux, d'une fidélisation du matelot à la compagnie ${ }^{8}$.

\section{Configurations du navire, déformations des rôles}

17 Six heures du matin, nous larguons les amarres tout de suite après avoir noté l'heure de fin de l'OC et signé les papiers commerciaux. En manœuvre, l'organisation du travail se transforme. La passerelle est soudainement pleine d'acteurs. Le temps de quitter le port 
et son chenal, un pilote monte à bord afin de donner les indications de route au matelot timonier. Temps de concentration intense, la manœuvre est aussi parfois un moment de convivialité où le capitaine et le pilote discutent, échangent nouvelles locales et informations diverses. Le pilote constitue alors un peu un lien entre le navire, espace clos et isolé, et la terre ferme que l'on n'a plus le temps de fouler tellement les opérations commerciales vont vite. Pendant ce temps, les deux autres lieutenants pont se trouvent aux plages de manœuvre avant et arrière, accompagnés chacun de trois matelots, afin de récupérer les amarres larguées par les lamaneurs depuis le quai. Enfin, les officiers mécaniciens sont en veille au PC machine : ils veillent à ce que tout se passe bien au niveau des installations, tout en se tenant prêt à intervenir rapidement si besoin. La manœuvre est en effet un moment critique où les risques sont plus nombreux qu'en pleine mer : les côtes sont plus proches et plus mesquines, le courant plus fort, le trafic plus dense. C'est pourquoi il importe que chaque personne tienne son poste jusqu'à la fin de la manœuvre.

Une fois le pilote débarqué, le navire passe en route libre. La configuration et l'activité se modifient, les rôles de chacun évoluent à nouveau.

En route libre donc, le travail à la passerelle consiste essentiellement en de la veille. Lorsque qu'elle est connue, la route, préparée par le lieutenant navigation, est entrée dans les outils d'aide à la navigation (GPS, radar) qui l'enregistrent et servent de référentiel pour les lieutenants. Il s'agit donc de veiller à ce que le navire suive le bon cap ( "la ligne rouge »), tout en étant attentif aux dangers potentiels : côtes, navires, vagues, vent, courants. Toutes les heures, le lieutenant fait le point sur la carte papier afin de témoigner de l'avancée du navire. Le quart est aussi un temps où les discussions sont possibles, car le travail de surveillance permet une certaine disponibilité de l'esprit. En ce sens, certains officiers mécaniciens diront qu'ils ont choisi la machine, "parce qu'au pont, on ne fait que pousser de l'eau. » En effet, tant que tout va bien, il y a peu de choses à faire hormis le point, éventuellement anticiper le prochain voyage, ou encore lire la nouvelle documentation. Mais parfois, un pic de stress perturbe la veille, ou même mieux, les officiers vont rechercher l'adrénaline.

Lorsque nous avons quitté l'Italie, le commandant n'avait aucune autre consigne que de descendre l'Adriatique afin de se tenir prêt à prendre toute destination en fonction des appels d'offre. Or le week-end s'annonçant, les bureaux du siège étaient en train de se vider de leurs employés, présageant deux jours et deux nuits sans nouvelles. La destination arriverait peut-être lundi matin, sûrement plus tard. ${ }^{9}$ Nous avions donc au minimum deux jours d'attente en prévision, à la dérive en pleine mer. Etre à la dérive n'est pas passionnant : il n'est pas possible d'engager de gros travaux à la machine car le navire doit être sur le qui-vive, et le travail à la passerelle consiste en repositionner régulièrement le navire en dérive dans une zone prédéfinie.

21 Afin d'optimiser ce temps d'attente, le commandant propose à la Zef de lui faire faire un exercice d'homme à la mer. L'exercice est peu souvent réalisé car il nécessite du temps et la collaboration d'une bonne partie de l'équipage: les matelots doivent aller chercher l'homme cobaye confectionné de bouées, le jeter à l'eau et rester à la poupe avec une perche afin de le récupérer, le lieutenant de quart et le commandant préparer l'exercice avec l'élève, et la machine se mettre en configuration de manœuvre. La coordination entre les services est primordiale, il importe de prévenir tout l'équipage car si de telles initiatives sont possibles, elles doivent néanmoins être réalisées en toute sécurité. Une fois validée la zone de l'exercice par le commandant, la zef se met alors à l'œuvre, jouant 
avec le joystick de la barre afin de faire virer le gros pétrolier. Tout le monde est attentif, certains mécanos sont même montés à la passerelle pour l'occasion afin d'observer la manœuvre. Premier essai, plutôt réussi, mais le navire passe quelques mètres trop loin de l'homme qui, du coup, est en train de se noyer. Le commandant relance alors l'élève et lui demande de recommencer. Ce qu'elle fait, or, en moins de cinq minutes, trois bateaux de pêche se retrouvent en route de collision avec le tanker. Le commandant demande de poursuivre la manœuvre, jusqu'au moment où la situation devient vraiment problématique, les pêcheurs ne comprenant pas à quoi s'essaie ce gros pétrolier.

Cet exemple montre à quel point, en quelques minutes, une situation apparemment très calme et sécurisée peut en quelques minutes devenir dangereuse. Ces moments sont sources de pics de stress pour les marins de quart qui, paradoxalement, attendent l'événement, voire, comme ici, le provoquent afin de briser la routine.

\section{Désorganisations du travail}

La vie à bord est donc rythmée par les heures de travail en route libre, et le rush des opérations commerciales. S'il est faux de dire que les marins se reposent en pleine mer, il faut toutefois insister sur la rupture que provoquent les temps à quai. Ces changements radicaux de rythme augmentent la fatigue de façon significative.
A bord du pétrolier, carnet de terrain
Chargement terminé à quatre heures du matin. Le second capitaine est donc sur le pied de guerre depuis trois heures, alors qu'il est allé se coucher vers 23 heures- minuit. De son côté, le lieutenant qui a terminé son quart au PC cargo à quatre heures, doit se relever à huit heures pour participer à la manœuvre qui dure une heure et demi, et ensuite réembaucher à douze heures pour son quart à la passerelle. De même pour son matelot qui fait le 12-18 heures à la coupée en OC, puis celui du minuit - six heures à la coupée, avec deux heures de repos avant la manœuvre à huit heures. De 9 h30 à midi, repos, puis réembauche pour travailler au pont jusqu'à seize heures, pause, et enfin quart à la passerelle de minuit à quatre heures du matin.

Si sur cette tranche de 24 heures, ce matelot a malgré tout douze heures non travaillées, le morcellement en plusieurs petits temps de repos et l'irrégularité du rythme de travail est source d'une grande fatigue.

Néanmoins, lorsque les rythmes se trouvent complètement décalés et que cela est possible selon le planning d'escale et les contraintes de la navigation, le capitaine peut décider de donner des demi-journées de congés après une escale.

A bord du vraquier, 370 mètres, transportant du minerai de fer, carnet de terrain

Nous sommes partis, direction le grand Nord. Le temps est compté car nous n'avons que trois jours et trois nuits de navigation pour nettoyer les neuf cales. En effet, le minerais du prochain chargement est d'une autre nature. Il ne faut donc pas mélanger les deux produits. Pour le nettoyage : des pelles, des gros seaux et ensuite un coup de jet d'eau. Nous descendons donc en fond de cale, éclairés par deux projecteurs, pour racler à la pelle les restes de minerais, les remonter à l'aide d'un treuil, et ensuite les jeter à l'eau. Après deux journées de travail exténuantes, les 
cales sont enfin propres. Il faudra encore passer le jet d'eau, mais cela est du ressort du Bosco. À la suite de cet épisode, n'ayant pas eu de vraies nuits pendant trois jours, les marins ont obtenus des demies journées de congés à tour de rôle. situation dus gros boulot, le service machine, après avoir jaugé les conditions de réalisation de ce job, prévient donc toujours le pont. Par ailleurs, cette planification reste étroitement dépendante de la zone de navigation du navire et de son planning d'escale.

Il y a 50 nœuds de vent depuis ce matin, beaucoup de roulis à cause d'une jolie houle qui nous rappelle la mer sous la ferraille. Ça fait du bien quelque part, même si la fatigue s'en trouve accentuée. L'activité est donc un peu ralentie, les gens fatigués, et puis tout simplement, il est compliqué de travailler avec du roulis d'au moins dix degrés. Les papiers tombent, les outils glissent, il est difficile de trouver son équilibre. Certains sont plus ou moins à l'aise, d'autres sont carrément malades.

Nous en sommes au troisième jour de dérive. Je demande au second capitaine s'il a reçu des informations et si je peux me rendre utile sur le pont. Celui-ci me répond qu'il ne sait pas, qu'il attend les nouvelles du port pour savoir quand nous allons pouvoir nous mettre à quai. D'ici là, difficile de planifier quoi que ce soit. Il ne faut toucher à rien avant le chargement parce que "si quelque chose pète, on est mal". De plus, en raison du mauvais temps, le port a fermé quelques heures. Il y a donc la queue à l'entrée du port, une dizaine de navires attend de pouvoir se mettre à quai afin de charger. Aussi, nous devons patienter le temps que la queue se tasse pour pouvoir aller à quai. Or vu la météo, ça s'annonce mal.

S'il est compliqué de planifier et d'engager de la grosse maintenance dans ce contexte, l'incertitude constante semble être cependant, dans une certaine mesure, appréciée. Elle casse la routine, ouvre la porte à la surprise, permet de spéculer sur le futur, oblige à se creuser la tête pour envisager différents scénarios de navigation.

Ici on fait tout au dernier moment. Mais ça marche, c'est ça qui me fait plaisir aussi. Je vois les bateaux qui allaient dans le Pacifique, on planifiait des trucs parfois on les faisait pas. Mais ici on fait tellement de choses, on s'arrête au port, et encore autre part, mais y'a toujours moyen de caser quelque chose, donc au final je trouve qu'on bosse mieux ici que sur les bateaux du Pacifique. Si c'est pas demain, ce sera après-demain. C'est bien pour ça. Mais faut être prêt. (Chef)

L'organisation de la maintenance nécessite donc une certaine flexibilité qui permet de prendre en compte les imprévus propres à la navigation. Le navire étant une entité indépendante pouvant vivre en autarcie quelques temps, il permet de penser une organisation du travail relativement autonome où les acteurs choisissent et composent en fonction de contraintes qu'ils vivent directement. 


\section{Traçabilités, responsabilités, sécurité}

30 Ces premières lignes montrent les différents types de contraintes auxquels est soumis le navire. Système socio-technique très complexe, il concentre en son sein un nombre élevé de risques qui tirent leurs origines de différentes sources (écologiques, techniques, humaines, etc.). Par ailleurs, la contrainte commerciale est très présente: le navire à l'arrêt est source de coûts élevés pour l'armateur dont l'objectif est, de ce fait, de faire naviguer ses navires, chargés, le plus possible. Assurer cette continuité du flux en dépit du turnover de la main d'œuvre nécessite de mettre en place des outils spécifiques afin d'assurer la sécurité des hommes et le bon fonctionnement des machines. Les passations de quart et les relèves d'équipage sont, dans ce cadre, des moments cruciaux car particulièrement sources de fragilités pour l'organisation. Enfin, tout ceci se déroule dans un cadre dominé par l'incertitude: incertitude économique du tramping, de l'environnement (mer, météorologie), de l'aléa humain. Les évolutions du travail à bord des navires depuis une vingtaine d'année témoignent ainsi d'une volonté d'apprivoisement de cet aléas, tout en poursuivant un objectif de plus de sécurité, sans pour autant bousculer le lobby économique.

Dans cette optique, une nouvelle réglementation internationale, le code ISM, a vu le jour en 2002 sous l'impulsion de l'organisation maritime internationale (OMI), agence spécialisée de l'ONU. L'ISM (International Safety Management) a pour objectif de formaliser le travail à bord à l'aide de la mise en place de standards internationaux, applicables à tous les navires de commerce au monde. L'ambition affichée est de réduire les imprévus du facteur humain, garantir la « sauvegarde de la vie humaine » et prévenir les risques de pollution. Il s'agit dès lors de développer une «culture de la sécurité » dans le milieu maritime.

Pour ce faire, le code se donne pour objectif de réguler et encadrer au mieux les gestes et processus de travail à l'aide de la mise en place de toute une documentation. Check lists, procédures, et rapports d'accidents ont ainsi fait leur entrée sur les navires grâce à un travail de décomposition et de description des tâches auquel les navigants ont été associés. Dans un second temps, ces éléments sont traduits par le service ISM de la compagnie en check lists et procédures et sont ensuite introduites sur le navire. Ce côté-ci du code, résumé par le credo : je fais ce que j'écris et j'écris ce que je fais, a été pensé afin de prévenir les accidents en réglant les actions effectuées par les acteurs, et/ou en cas d'accident, de pouvoir déterminer la cause de celui-ci grâce à la traçabilité écrite des actions effectuées et à la signature des opérateurs.

L'autre dimension du code tend à faciliter la communication entre le bord et la terre afin de faire remonter les anomalies, les problèmes plus ou moins ponctuels auxquels la compagnie doit apporter une réponse, ou, en sens inverse, de faire entrer une nouvelle réglementation extérieure au navire. Face aux retours d'expérience, comptes rendus, et rapports d'évaluation des risques rédigés par les navigants, l'armateur a un devoir de réponse. En marche normale, ce nouveau système organisationnel basé sur l'écrit doit donc s'autoréguler et s'autoalimenter en vue d'une amélioration constante de l'organisation du travail et, partant, de la réduction des risques et accidents.

34 Cependant, si les objectifs visés par le code font consensus (qui souhaite qu'une marée noire se produise le long de ses côtes ?!), force est de constater que dans l'activité, son utilisation est handicapante. Les enjeux que soulève ce code dépassent en effet de loin la 
candide volonté d'inculquer une « culture de la sécurité » auprès de marins qui n'auraient aucune idée des risques auxquels ils sont soumis et qu'ils sont susceptibles de provoquer.

Pour un boulot par exemple, de soudure. Si vraiment on s'en tient à la lettre, même si c'est un truc urgent, faut faire un permis de travail à chaud par exemple, et qu'on le submit à Paris, qu'on attende la réponse comme quoi ils sont d'accord. Je ne vois pas dans quelle condition, même avec le papier, eux, ils peuvent juger que oui...ça, ça devrait être un truc qui devrait rester à bord. C'est encore une couverture au cas où... Mais quand on fait un truc comme ça, on est conscient de ce qu'on fait. On va pas aller souder sur le pont, ou aller fumer sur le pont. ( $2^{\text {nd }}$ mécanicien)

De manière générale, le caractère infantilisant et chronophage des documents est ici d'autant plus mis en cause, que, souvent, ces derniers viennent concurrencer d'autres types d'écrits ${ }^{10}$, et, finalement, entravent l'activité. Par exemple, quelques jours après notre départ, le second capitaine s'est rendu compte que le navire faisait de l'eau. Selon le code ISM, celui-ci doit descendre dans les ballasts accompagné d'une ou deux personnes, et pour chacune d'entre elles, il doit rédiger un permis de travail en espace clos, lequel doit être validé par la compagnie avant de pouvoir enfin descendre. Bien sûr, le second n'a pas pris le temps de faire ces permis, car ceci aurait pris une heure et demie de rédaction, alors que les marins sont a priori aptes à descendre dans de tels espaces et, qu'au nom de la sécurité, la priorité a été donnée à l'action de descendre afin de trouver au plus vite la fuite. Dans cette situation, les documents, rédigés après coup, ne sont donc pas légalement valables. Mais, antidatés, ils seront le gage papier que la procédure a été bien suivie.

Ça, c'est parce que la compagnie veut continuer à travailler avec les grands du pétrole à moindre coût, surtout avec la conjoncture actuelle. Du coup il faut que tout soit nickel, sur le papier du moins. Parce qu'après entre ce qu'on nous demande de faire et ce qu'on fait vraiment, il y a un gouffre.

Plus largement, le fonctionnement du code, justifié par son objectif d'autorégulation, remet en cause l'expertise des marins. À travers cette uniformisation des pratiques, les check lists et les procédures nient tous savoirs et savoir-faire "de la place » ${ }^{11}$, ces ingéniosités et arrangements du quotidien, en ouvrant un espace plus large à l'expertise externe de la terre. Le problème est que justement, le cœur du métier des navigants, est de savoir se débrouiller avec les moyens du bord, de façon autonome grâce à des connaissances et savoir-faire très polyvalents.

C'est-à-dire qu'on a un problème, nous on dit qu'on n'a jamais de problème, mais que des solutions. L'état d'esprit c'est d'avoir tout le temps une ou deux solutions derrière. Parce qu'on est tous seuls, faut que ça marche, y'a un moment où de toute façon y'a que toi qui peux gérer le truc, et puis moi je me dis, tu fais tout pour que ça marche, et puis si ça marche pas, y'a un moment où tu es au bout de tes compétences. Et puis on travaille en équipe, on échange. C'est ça qui fait la force du marin. C'est le fait que quand on est en mer, on est tout seul donc on prend cette habitude de chercher une ou plusieurs solutions au problème qu'on a. C'est clairement la grosse différence de notre métier.

La logique disciplinaire de l'ISM entre en contradiction avec la culture professionnelle des navigants ${ }^{12}$ d'autant plus violemment qu'elle tire ses origines d'un extérieur qui se pose en expert de la sécurité. Le code ISM procède donc bien à une aliénation au sens strict du terme : son utilisation suppose une certaine dépossession du monopole d'un savoir-faire porté par les marins. Il décentralise l'expertise du navire vers la terre. Cette perte d'autonomie accentue un sentiment de dévalorisation de leur activité, que traduit la sensation souvent exprimée de n'être aujourd'hui plus que des "routiers". Plus généralement, ce débat fait apparaître la construction de la sécurité dans ce qu'elle a de 
plus sociale. Comme le montre L.M. Barnier pour l'aviation ${ }^{13}$, celle-ci résulte d'une opération de valorisation et reconnaissance d'une certaine manière de "faire " la sécurité, s'imposant comme étant la plus efficace, la plus rationnelle. En cela, l'ISM participe à la distribution des «formes d'intelligences socialement acceptables et socialement acceptées $»^{14}$. Ce qui est ici en jeu, est bien le conflit entre deux logiques, deux mondes sociaux: celui des marins qui refusent de voir la terre substituer leur savoir-faire de praticien à celui de l'ISM, et celui de la terre qui entend faire passer dans le navire ses propres contraintes.

Cette ouverture sur l'activité ne fait pas toujours sens pour les marins de l'entreprise qui venait tout juste de se faire racheter par un géant du transport maritime. Lors de cet achat fusion, les rôles ont été redistribués, et les volontés de regard multipliées : selon la question, le domaine concerné, le bord a dorénavant affaire à un bureau situé soit à Singapour, soit au Danemark, soit en France :

C'est devenu tellement énorme qu'ils sont obligés de mettre en place des systèmes, des procédures pour n'importe quoi, même pour discuter! Je pense c'est aussi au niveau culturel, ils embauchent tellement de gens de cultures différentes, de nationalités différentes qu'ils sont obligés de normaliser la manière dont se font les échanges, la manière dont vont se faire les choses

Il faut rendre des comptes à davantage d'entités, qui ont toutes un droit de regard sur ce qui est réalisé à bord, et comment cela est fait. L'écrit apparait alors ici comme un palliatif à l'éclatement des unités de décision de la compagnie. Ouvert sur le monde, mais en même temps espace complètement clos, le navire est l'objet de volontés de contrôle aux multiples sources.

Pour autant, on n'est pas contre écrire. Ecrire, c'est bien. Même le code ISM c'est bien, au début du moins, ça part d'une bonne intention. C'est la dérive qui va avec qui va pas, c'est trop. Les procédures par exemple, tu as eu une journée éprouvante, tu es en manœuvre le soir, jusqu'à trois heures du matin. Tu t'endors. On te réveille, tu n'es pas frais. Donc qu'est ce que tu vas faire? Tu vas prendre la procédure pour vérifier que tu n'as rien oublié. Pour ça, c'est bien. De même, quand tu arrives sur un bateau que tu ne connais pas, tu vas regarder la doc pour découvrir un peu le truc. Si la doc y répond pas, tu vas demander, et puis voilà.

40 Ce qui semble être vraiment mis en cause par les marins n'est donc pas l'effort que l'action d'écrire suppose, ni la présence de documents jouant le rôle d' "artefacts cognitifs $»^{15}$ dans l'activité. Les marins développent eux-mêmes des outils scripturaires pour faciliter leur travail, et la documentation est d'autant plus utile que le turnover entre les différentes compagnies et navires d'une même compagnie est important. Ce qui semble donc poser problème est plutôt la dimension juridique de ces documents. Lorsqu'ils écrivent, en signant en bas de la feuille, les marins s'engagent nominativement. Ces écrits deviennent alors autant de pièces à conviction lors d'un accident ou d'une avarie pouvant mettre en cause individuellement le marin. Ceci renforce le sentiment selon lequel la documentation sert avant tout la compagnie qui se dote par ce biais d'une "vitrine" propre pour ses clients, tout en se déresponsabilisant. Alors qu'une des caractéristiques de l'expédition maritime était la mutualisation des risques, les documents ISM semblent ramener la responsabilité au plus proche du lieu où le risque a lieu tout en individualisant la prise de risque.

41 Pour faire face à cette tendance, les stratégies sont nombreuses.

C'est devenu absurde car par exemple, quand le second veut faire une commande de matériel, argumentée comme le veut la boîte, tout doit passer par moi quand même, donc si c'est cinquante lignes par matériel, tu y passes la nuit. Donc moi c'est niet, je 
veux pas tomber malade à cause du travail, donc quand je monte dans ma cabine, c'est off, je regarde plus mes mails passés cette heure-là. Pareil pour le matin, je me refuse à arriver à huit heures à la machine et à regarder directement mes mails. (Chef)

En dépit de l'introduction d'internet, l'opérateur a en effet toujours la possibilité de retarder l'allumage de son ordinateur... Mais plus encore, celui-ci peut refuser d'écrire et choisir de faire sans en rendre compte par écrit, ou bien d'écrire sans faire. Les officiers sont ainsi amenés à "tricher» quotidiennement afin de mettre le papier en conformité avec ce que les acteurs extérieurs au navire souhaitent que la réalité soit. Or la réalité à bord, faite d'incertitudes et d'imprévus est souvent éloignée de ce monde de papier.

\section{Du temps pour soi}

Le travail à bord des navires nécessite ainsi une attention de tout instant, ainsi qu'une disponibilité totale du travailleur au service de son travail. Cette mise à disposition de soi appelle, dans la mesure du possible, à l'aménagement d'espaces et de temps pour soi. Le navire comporte en effet toutes les caractéristiques de l'institution totale de Goffman comme « lieu de résidence et de travail où un grand nombre d'individus, placés dans la même situation, coupés du monde extérieur pour une période relativement longue, mènent ensemble une vie recluse dont les modalités sont explicitement et minutieusement réglées. $~^{16}$

Cependant, malgré le - ou à cause du - caractère très totalisant de cette institution, les moments témoins de prises de distance avec cette dernière ne sont pas rares. Le repli sur son unique espace privatif, la cabine, seul lieu qui permet un tant soit peu d'intimité, les soirées karaoké du samedi soir, ou encore les moments de détente où l'on sort les cannes à pêche sont autant de manifestations de la volonté de se faire un quotidien agréable en dépit de cette veille constante au service du travail. Car si les officiers français disposent de temps de congés conséquents, les matelots passent souvent plus de temps en mer que chez eux.

Pour les officiers, cette prise de distance avec l'organisation se manifeste surtout par la coupure radicale savamment entretenue entre les congés et les temps embarqués. Nombreux sont ceux qui ne pratiquent aucune activité maritime lorsqu'ils sont à terre, et disent ne pas vouloir entendre parler de bateau, de compagnie et de collègues pendant leurs congés. Cette distinction entre les deux sphères parle d'elle-même. Il est compréhensible qu'au sortir de deux ou trois mois d'embarquement où le corps et l'esprit ont été entièrement tournés vers le travail, le marin cherche à changer radicalement de quotidien. En mer, la rupture avec le monde est telle qu'elle appelle à une autre rupture tout aussi radicale au retour, laquelle est rendue possible par l'organisation même de l'acte productif. Une fois débarqué, le marin ne peut rien faire ou presque pour le navire qui est alors pris en charge par la relève.

La situation est très différente pour les matelots qui vivent souvent plus longtemps sur le navire qu'au sein de leur famille. Les fenêtres de temps libres sont d'autant plus importantes à aménager qu'il s'agit pour eux de se ménager afin de durer dans le temps, d'économiser ses forces. Les cabines de matelots sont ainsi plus souvent investies que celles des officiers. 
47 Le quotidien s'organise donc entre les heures de travail pures et les heures de repos, rythmant les semaines et les jours qui se ressemblent beaucoup. Dans ce huit clos, les possibilités d'exit sont drastiquement limitées. Avec les réformes portuaires, l'éloignement des terminaux de tout centre urbain, l'accélération des opérations commerciales, le nombre restreint de membres d'équipage et le renforcement du contrôle de la mobilité des marins découlant de l'adoption de lois antiterroristes, les escales se font de plus en plus exceptionnelles. Néanmoins, lorsque c'est possible, sortir fouler la terre ferme constitue un temps à part, où l'on s'extirpe pour quelques heures de sa place assignée à bord. Pour la sortie, les vêtements « civils » sont ressortis de la valise, les bleus, shorts et tongs laissés au vestiaire. Les escales sont par ailleurs des moments plus propices pour parler des choses personnelles. Alors qu'à bord, la retenue est constante, qu'il faut faire attention à se taire quand il faut tout en parlant suffisamment, les langues se délient un peu plus une fois quitté le navire. Pendant ces quelques heures d'escales, les masques tombent donc, un peu.

Cependant, les escales ne sont pas les seuls moments de convivialités et de prise de distance avec l'organisation. Au fur et à mesure que la côte se fait plus petite à l'horizon, les pressions des divers acteurs s'atténuent. L'éloignement des côtes permet de réaffirmer la clôture autour du navire ainsi que l'autonomie qui le caractérise. Le capitaine redevient un temps maitre à bord pour organiser le travail, en dépit des dizaines de mails qu'il reçoit quotidiennement. J'ai ainsi pu voir un capitaine arriver en fin de journée au carré en brandissant un mail reçu de la part du service commercial de l'armateur, rouge de colère. Ce document livrait des calculs de navigation pour le prochain voyage, alors même que celui en cours venait tout juste de commencer. Devant l'absurdité de la situation, le commandant remet le papier dans sa poche arrière en affirmant que de toute façon il ne répondrait pas à ce mail avant le lendemain matin. Il faut soulever ici les enjeux de cette situation: par ce mail, le service commercial communique au commandant des calculs effectués habituellement par les officiers afin de préparer l'escale : tirant d'eau, tirant d'air, stabilisation selon la charge du navire. Par l'envoi de ce mail, le service commercial empiète littéralement sur la division du travail entre les marins, en charge d'organiser la navigation et les OC, et le service commercial, dont le rôle est, notamment, de trouver des appels d'offre. En décidant de ne pas répondre à ce mail, le commandant brave ainsi l'autorité de son armateur, publiquement, alors même qu'il en est le représentant légal et symbolique à bord.

Ce faisant, le commandant réaffirme par ailleurs son expertise tout en redéfinissant les rôles de chacun. Il n'est par ailleurs pas anodin que les moments collectifs les plus marquants se déroulent en plein océan Atlantique où les navigants prennent le temps de faire des barbecues. Ceci se fait bien sûr pour des questions de sécurité, mais il me semble aussi que loin des ports et des bureaux, il est plus aisé d'affirmer son rôle de marin. Car en dépit de tous les outils de contrôle et de communication établissant un lien direct entre la terre et le navire, les marins sont encore ceux qui occupent le navire et le font marcher.

\section{Do It Yourself !}

L'organisation du travail à bord d'un navire de commerce est donc très particulière. Fonctionnant en autonomie totale, soumis à un certain nombre de contraintes, et devant répondre à l'impératif de sécurité, il est important de définir les rôles de chacun. À bord, chacun sait ce qu'il a à faire, selon la configuration et la situation du navire. 
51 Lieu de travail mais aussi lieu de vie, le marin embarqué doit se mettre à disposition de l'activité. Y compris pendant ses temps de repos à bord, et en raison d'effectifs à bord trop réduits, il importe d'être disponible à tout moment pour répondre à l'aléa. Les imprévus sont nombreux, et il s'agit de s'organiser pour y faire face afin d'assurer le bon déroulement de l'expédition commerciale. Dans ce cadre, une des particularités du métier est donc de se débrouiller avec les moyens du bord. Cette donnée est centrale car elle est constitutive de l'identité professionnelle. Le navire fonctionne en cercle fermé. En mer, pas de service de maintenance spécialisée. Il faut bricoler et s'arranger comme on peut.

Les récentes évolutions du travail à bord remettent en cause cette clôture du navire mais de façon paradoxale. S'il est toujours demandé aux marins de se débrouiller avec les moyens du bord, la terre s'invite comme experte extérieure au navire et participe ainsi au développement d'un sentiment de dépossession du savoir faire des marins. Le code ISM semble supposer que dorénavant, pour être un bon marin, il suffit de savoir suivre une procédure ou une check list. Or la réalité est bien plus complexe qu'une suite d'items et nécessite toujours de mobiliser son expérience et la réflexion collective. Or si tout le monde semble être d'accord sur ce point, ce qui fait dorénavant foi est ce qui apparaît sur le papier.

53 Ceci invite à pousser la réflexion plus avant pour mettre en évidence les enjeux de responsabilité que ces nouvelles politiques de management, non spécifiques au maritime, soulèvent. Dans un monde où tout un chacun cherche à se dédouaner, et surtout les plus puissants, il s'agit de construire des chaînes de déresponsabilisations en repoussant la faute au plus près du lieu où le risque est pris. Aussi, si le siècle précédent a été le théâtre d'une mise en place d'une mutualisation des risques ${ }^{17}$, il apparaît que, et l'exemple du maritime l'illustre parfaitement, nous soyons sur un chemin inverse qui tend à individualiser la prise de risque.

54 Cette évolution est forte de sens pour un secteur où la mise à disposition de soi au service de l'activité est déjà très présente. Les stratégies pour se détourner de cette tendance sont donc nombreuses et se manifestent, on l'a vu sous différentes formes. Néanmoins, une des plus marquantes - en partie aussi parce qu'elle est menée par le commandant lui-même qui, il n'est pas inutile de le rappeler, est le représentant de l'armateur à bord -, reste cette volonté de « re-clore » le navire afin de reconstituer l'autonomie qui le caractérise. Ceci constitue en effet une tactique forte afin de rappeler qu'en dépit des multiples outils de contrôle à distance de l'activité, n'étant pas présent à bord, l'armateur ne peut influer complètement sur la façon dont le travail se fait. Ce rappel est particulièrement intéressant dans cette organisation du travail où la marchandise, enjeu central de l'activité, est littéralement confiée aux salariés, lesquels possèdent entre leurs mains un excellent moyen de pression en cas de conflit.

\section{BIBLIOGRAPHIE}

BALIBAR E., CHEMILLIER-GENDREAU M., COSTA-LASCOUX J., TERRAY E. (1999), Sans-papiers :

l'archaïsme fatal, Paris, La Découverte 
BARNIER L.M., « La sécurité dans le transport aérien : un concept social », Colloque HIAS Human Issues in Aviation System 'Change, Cost, Reality', Septembre 2001

CHARASSE David, « L'usine, l'écriture et la place. Savoirs techniques dans les transports sidérurgiques (1954-1989) ", Genèses 7, mars 1992, p.63-93

EWALD F. (1986), L'Etat providence, Paris, Grasset

GOFFMAN E. (1968), Asiles. Etudes sur la condition sociale des malades mentaux. Paris, Editions de Minuit

JOUNIN N. (2008), Chantier interdit au public, Paris, La Découverte

NORMAN, D., « Les artefacts cognitifs », Raisons Pratiques, n 4, 1993, p. 15-34

VINCENT J.M., (1995), « La légende du travail », in La liberté du travail, COURS-SALIES (coord.), Syllepse, p71-82.

« Nouveau capitalisme et expertise quotidienne » Entretien avec Richard Sennett, Cahiers internationaux de sociologie, 2009/1 n¹26 p13-20

\section{NOTES}

1. L'affréteur est le propriétaire de la marchandise transportée, tandis que l'armateur est le propriétaire du navire qui loue ses services au premier. Ce dernier est également chargé d'armer le navire en hommes et biens, ainsi que d'assurer la maintenance des installations et machines du bord.

2. La majorité des entretiens a été réalisée à bord avec des officiers et des matelots. Ils sont insérés dans le texte en italique ou mis en exergue afin de faciliter la lecture. Les propos mis à la première personne sont issus de mon carnet de terrain dans lequel j'écrivais quotidiennement.

3. Pour International Ship and Port Security. Ce code a été mis en place à la suite des attentats du 11 septembre 2001 à New York.

4. Balibar E., Chemillier-Gendreau M., Costa-Lascoux J., Terray E. (1999), Sans-papiers: l'archaïsme fatal, Paris, La Découverte

5. L'ethnicisation des relations de travail existe également comme le montre N. Jounin pour le secteur du bâtiment en France (Jounin N. (2008), Chantier interdit au public, Paris, La Découverte). Cependant, il n'est pas rare de voir des navires avec des équipages composés de plus de trois nationalités différentes. À bord du pétrolier, il y avait trois nationalités : française, bulgare, et lettone. Mais chez les Lettons, il fallait distinguer les non citoyens lettons, en majorité issus de familles russes ayant migré en Lettonie qui constituent le bas de la hiérarchie sociale, des citoyens lettons.

6. Les navires sont soumis à différentes instances de contrôle : par les armateurs par des audits internes, par les administrations (en France, les affaires maritimes), par l'état du port où le navire fait escale (le Port State Contrôle), et enfin par les clients (audits externes)

7. Normalement. Car les cas de marins abandonnés par leur armateurs sont nombreux, et tout près de chez nous comme les marins de l'Antigone $Z$ qui se sont mis en grève le 3 avril 2012 à Douarnenez pour non paiement des salaires, ou encore ceux du RSV Endeavour à Brest en grève depuis 24 mai 2012.

8. Pour toutes ces questions de « convocabilité » du travailleur face à leurs agences de gestion de travail, voir CHAUVIN S., Les agences de la précarité. Journaliers à Chicago, Paris, Seuil, coll. «Liber », 2010, 339 p.

9. "On sait quand on embarque mais on ne sait jamais quand ni où", m'explique le $2^{\text {nd }}$ capitaine. Pour le chef, il faut que ce soit Milazzo, en Sicile, pour qu'il puisse enfin débarquer. Cela fait trois 
semaines qu'il est en temps supplémentaire de son contrat. "C'est le risque du métier", me dit il avec un sourire forcé. Si nous n'allons pas à Milazzo, cela signifie encore le week-end d'attente, puis le temps que l'armateur se saisisse d'un appel d'offre, les jours de navigation pour rejoindre la prochaine destination et enfin l'arrivée de la relève. Encore une semaine environ.

10. Journal passerelle et journal machine, et tous types d'écrits personnels

11. CHARASSE David, «L'usine, l'écriture et la place. Savoirs techniques dans les transports sidérurgiques (1954-1989) ", Genèses 7, mars 1992, p.63-93

12. CHARASSE David, «L'usine, l'écriture et la place. Savoirs techniques dans les transports sidérurgiques (1954-1989) », Genèses 7, mars 1992, p.63-93

13. BARNIER L.M., «La sécurité dans le transport aérien : un concept social ", Colloque HIAS Human Issues in Aviation System 'Change, Cost, Reality', Septembre 2001

14. VINCENT J.M., (1995), « La légende du travail », in La liberté du travail, COURS-SALIES (coord.), Syllepse, p71-82. Voir également « Nouveau capitalisme et expertise quotidienne » Entretien avec Richard Sennett, Cahiers internationaux de sociologie, 2009/1 n¹26 p13-20

15. NORMAN, D., « Les artefacts cognitifs », Raisons Pratiques, $n^{\circ}$ 4, 1993, p. 15-34

16. Goffman E. (1968), Asiles. Etudes sur la condition sociale des malades mentaux. Paris, Editions de Minuit (p.41)

17. EWALD F. (1986), L'Etat providence, Paris, Grasset

\section{RÉSUMÉS}

Il est surprenant de constater qu'en dépit de la centralité du transport maritime dans nos quotidiens, ce secteur reste aujourd'hui si largement méconnu. D'un premier abord, s'intéresser de près au fonctionnement du secteur de la marine marchande, fait l'effet d'un coup de massue. L'organisation du travail, la gestion de la main d'œuvre, les acteurs jouant sur cette scène, donnent l'impression d'une machine de guerre capitaliste que rien ne peut arrêter. Les discours fatalistes à son égard, de la part des acteurs eux-mêmes, sont monnaie courante. Si les marins n'arrivent pas à faire respecter leurs droits fondamentaux, s'il arrive souvent que les salaires ne sont pas versés, s'il est normal que sur un même navire plusieurs statuts et droits du travail différents s'appliquent, c'est que le maritime fonctionne ainsi, un point c'est tout. Recouvrant une certaine réalité, ce discours bien lisse est cependant éloigné d'une réalité beaucoup plus complexe : ici comme ailleurs, les acteurs sont à l'origine de tactiques afin de se détourner d'une organisation qui se veut totalisante.

Cet article, sorte de patchwork d'idées en cours, se veut volontairement un texte non problématisé, dont le point de départ sont les entretiens effectués avec des marins de commerce et les éléments de terrain récoltés lors d'embarquements réalisés au cours des deux années passées. Il s'agit donc avant tout de faire connaître un peu plus l'organisation d'un secteur vivant dans une discrétion bien utile, afin d'amorcer des réflexions plus approfondies et généralisables à d'autres secteurs. 
INDEX

Mots-clés : critique du travail, correspondance, artistes, socialisme vert, travailleurs culturels, Wertkritik, Gorz André, dialogue, Vincent Jean-Marie, écologie, modèles alternatifs, Marx Karl, penser le Capital, ethos du travail, Eglise Orthodoxe, salariat, domination

\section{AUTEUR}

\section{CLAIRE FLÉCHER}

Doctorante en sociologie, Université Paris X Nanterre. 